\title{
On the Approximation of Blow-up Time for Solutions of Nonlinear Parabolic Equations
}

By

\author{
Takeo K. UshiJimA*
}

\begin{abstract}
There are many nonlinear parabolic equations whose solutions develop singularity in finite time, say $T$. In many cases, a certain norm of the solution tends to infinity as time $t$ approaches $T$. Such a phenomenon is called blow-up, and $T$ is called the blow-up time. This paper is concerned with approximation of blow-up phenomena in nonlinear parabolic equations. For numerical computations or for other reasons, we often have to deal with approximate equations. But it is usually not at all clear if such wild phenomena as blow-up can be well reflected in the approximate equations. In this paper we present rather simple but general sufficient conditions which guarantee that the blow-up time for the original equation is well approximated by that for approximate equations. We will then apply our result to various examples.
\end{abstract}

\section{$\S 1$. Introduction and Main Results}

There is a large number of nonlinear partial differential equations of parabolic type whose solution for a given initial data cannot be extended globally in time and develop a singularity in finite time, say $T$. Such a phenomenon is called blow-up and $T$ is called the blow-up time. In many cases, some norms of blow-up solutions tend to infinity as $t$ approaches $T$.

Blow-up is known to occur in various equations including those in combustion theory, chemotaxis models and equations describing crystalline formation involving curvature-driven motion (see [9], [39j). The study of blow-up phenomena is not only interesting from the mathematical point of view but also

Communicated by H. Okamoto, March 9, 2000. Revised April 20, 2000.

1991 Mathematics Subject Classification(s): 65M12, 35K55

* Meme Media Laboratory (VBL), Faculty of Engineering, Hokkaido University, Kita 13,

Nishi 8, Kita-ku, Sapporo 060-8628, Japan.

e-mail: ushijima@aurora.es.hokudai.ac.jp 
important for deep understanding of the nature of the phenomena which those equations describe.

Since the appearance of the pioneering work of Fujita [18], blow-up phenomena in nonlinear parabolic equations have been studied extensively. Particular emphasis has been placed on the question as to when, where and how the solutions blow up. Nowadays rather precise information about blow-up solutions near the blow-up time is derived for some particular equations (see [31], [37], [42], and references therein). However, there are many open questions concerning blow-up.

Generally speaking, it is very difficult to numerically simulate blow-up phenomena accurately. For one thing, one has to deal with numerical data that grow indefinitely as the blow-up time approaches. This is obviously not an easy task. Secondly - and more importantly - it is not at all clear if features of such a wild phenomenon as blow-up can be well reflected in the discretized equation which approximate the original equation. Most of the standard error estimates become useless as $t$ approaches the blow-up time.

There have been some attempts to establish numerical methods to capture blow-up phenomena. For example, the rescaling algorithm by Berger and Kohn [10] and the method of MMPDE [12] by Budd et al can observe the shape of blow-up solutions near the blow-up time. There are also some works concerning approximation of blow-up time. Some authors studied numerical blow-up time and its convergence. As for the semilinear parabolic equation $u_{t}=u_{x x}+u^{p}$, Nakagawa [35] $(p=2)$ and Chen [13] $(p>1)$ studied a finite difference scheme for this equation and proved the convergence of the blow-up time of approximate solutions to that of the real solution. They assumed that $L^{q}(q=1$ or 2$)$ norm of the solution diverges at blow-up time. This assumption, however, does not always hold [17]. Recently, Abia, López-Marcos and Martínez [1] considered a one dimensional semi-discrete problem for this equation. Here a semi-discrete problem means the system of ordinary differential equations which is obtained by discretizing the space variable (but not the time variable) via finite difference approximation. They proved the convergence of blow-up time, on the condition that the $L^{\infty}$ norm of the solution blows up.

In this paper, we discuss the approximation of blow-up time in a rather general framework and establish simple sufficient conditions that guarantee the convergence of blow-up time. We will then apply these general results to various examples.

Now let us consider the initial-boundary value problem for the following 
nonlinear parabolic partial differential equation:

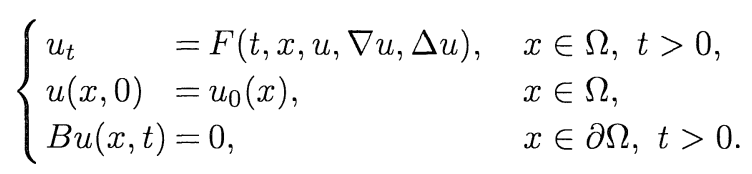

Here $B$ represents the boundary condition. We assume that this problem possesses a unique local solution $u(\cdot, t)$ in a function space $X$ and there exists finite time $T$ such that this solution cannot be extended in $X$ beyond $T$. Namely, we assume that

$$
\text { the solution } u(\cdot, t) \text { of }(1.1) \text { blows up at } T \text {. }
$$

Moreover, we assume that there exists a functional $J: X \rightarrow \mathbb{R}$ such that for the blow-up solution $u$ of (1.1) one of the following holds:

$$
\lim _{t \rightarrow T} J[u](t)=\infty,
$$

or

$$
\lim _{t \rightarrow T} \frac{d}{d t} J[u](t)=\infty .
$$

We note that if the condition (b) holds then there exists a function $H(t)$ such that

$$
\frac{d}{d t} J[u](t) \geq H(t), \quad \lim _{t \rightarrow T} H(t)=\infty .
$$

Various examples of such a functional $J$ can be found in many blow-up problems. Some of them will be given in Sections 3 below.

We approximate problem (1.1) by a family of equations which is parameterized by $h$ :

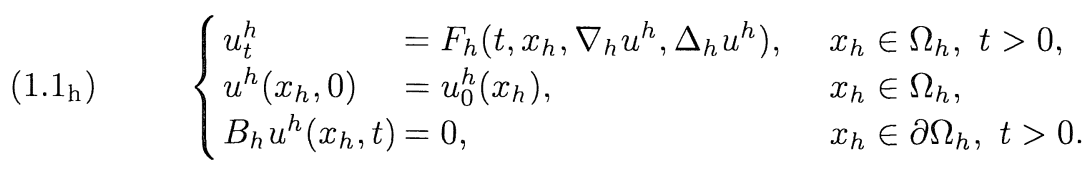

Here the parameter $h$ indicates the accuracy of approximation, which becomes better as $h$ tends to zero. The approximations of $F, B, \nabla, \Delta, \Omega, x$, and $u_{0}$ are denoted by $F_{h}, B_{h}, \nabla_{h}, \Delta_{h}, \Omega_{h}, x_{h}$, and $u_{0}^{h}$, respectively. The semi-discrete problem for $(1.1)$ is an example of $\left(1.1_{\mathrm{h}}\right)$. In this case, the parameter $h$ corresponds to the spatial mesh size. We assume that the approximate problem $\left(1.1_{h}\right)$ possesses a unique local solution in a function space $X_{h}$. Moreover, we assume that 
there exists a functional $J_{h}: X_{h} \rightarrow \mathbb{R}$ which approximate the above functional $J$.

The main result of this paper states that if the solution $u^{h}$ of $\left(1.1_{\mathrm{h}}\right)$ converges to the solution $u$ of (1.1) in the sense of functional (assumption (A2) below) and if the functionals $J$ and $J_{h}$ satisfy some conditions (one of assumptions (A1), $\left(\mathrm{A} 1^{\prime}\right),\left(\mathrm{A} 1^{\prime \prime}\right),\left(\mathrm{A} 1^{\prime \prime \prime}\right)$ below) then the following holds:

(c) the approximate solution $u^{h}$ blows up in finite time $T_{h}$ for sufficiently small $h$ and $T_{h}$ converges to $T$ as $h$ tends to zero.

Now we explain the conditions which should be satisfied by the functionals $J$ and $J_{h}$. Let us introduce two differential inequalities. The first is of the form

$$
\frac{d}{d t} I(t) \geq G(I)
$$

where $G:[0, \infty) \rightarrow \mathbb{R}$ is a function satisfying

$$
\left\{\begin{array}{c}
G(s)>0 \quad \text { for } s>R_{0} \\
\int_{R_{0}}^{\infty} \frac{d s}{G(s)}<\infty
\end{array}\right.
$$

The second is

$$
\frac{d^{2}}{d t^{2}} I(t) \geq-c \frac{d}{d t} I(t)
$$

where $c$ is a non-negative constant.

Let $u(\cdot, t) \in X$ be a given solution of (1.1) that blows up at $t=T$ and let $u^{h}(\cdot, t) \in X_{h}$ be a solution of $\left(1.1_{\mathrm{h}}\right)$ for each $h$. We assume that there exist a functional $J: X \rightarrow \mathbb{R}$ and a family of functionals $J_{h}: X_{h} \rightarrow \mathbb{R}$ such that the functions $J(t)=J(u(\cdot, t))$ and $J_{h}(t)=J_{h}\left(u^{h}(\cdot, t)\right)$ satisfy one of the following conditions:

(A1) $J(t)$ is a continuous function satisfying the condition (a), while $J_{h}(t)$ is a $C^{1}$ function satisfying the inequality (I1) for some function $G$.

$\left(\mathrm{A} 1^{\prime}\right) \quad J(t)$ is a $C^{1}$ function satisfying the condition (b), while $J_{h}(t)$ is a $C^{2}$ function satisfying the inequality (I2) for some non-negative constant $c$. Moreover, there exists a finite constant $M$ such that $J(t) \leq M$ and $J_{h}(t) \leq M$ for all $h>0$.

$\left(\mathrm{A}^{\prime \prime}\right) J(t)$ is a $C^{1}$ function satisfying the condition (b), while $J_{h}(t)$ is a $C^{2}$ function satisfying both the inequalities (I1) and (I2) for some function $G$ and a non-negative constant $c$. 
$\left(\mathrm{A} 1^{\prime \prime \prime}\right) J(t)$ is a $C^{1}$ function satisfying the condition $\left(\mathrm{b}^{\prime}\right)$ for a function $H$, while $J_{h}(t)$ is a $C^{1}$ function satisfying the inequality (I1) for a function $G$ and inequality

$$
\frac{d}{d t} J_{h}(t) \geq H_{h}(t)
$$

for a function $H_{h}(t)$ which satisfies $\frac{d}{d t} H_{h}(t) \geq 0$.

We also assume one of the following.

(A2) The family $u^{h}$ approximates $u$ in the following sense: for any $\epsilon>0$,

$$
\lim _{h \rightarrow 0} \sup _{t \in[0, T-\epsilon]}\left|J[u](t)-J_{h}\left[u^{h}\right](t)\right|=0 .
$$

$\left(\mathrm{A} 2^{\prime}\right)$ The condition (A2) holds and for any $\epsilon>0$,

$$
\lim _{h \rightarrow 0} \sup _{t \in[0, T-\epsilon]}\left|H(t)-H_{h}(t)\right|=0 .
$$

Our main results are the next four theorems.

Theorem 1.1. Assume (A0), (A1), and (A2) then (C) holds.

Theorem 1.2. Assume (A0), (A1'), and (A2) then (C) holds.

Theorem 1.3. Assume (A0), $\left(\mathrm{A}^{\prime \prime}\right)$, and (A2) then $(\mathrm{C})$ holds.

Theorem 1.4. Assume (A0), ( $\left.\mathrm{A}^{\prime \prime \prime}\right)$, and $\left(\mathrm{A} 2^{\prime}\right)$ then $(\mathrm{C})$ holds.

Our main results assert that if the original problem has a functional $J$ which satisfies $(\mathrm{A} 1)\left(\left(\mathrm{A} 1^{\prime}\right),\left(\mathrm{A} 1^{\prime \prime}\right)\right.$, or $\left.\left(\mathrm{A} 1^{\prime \prime \prime}\right)\right)$ and if the approximate problem well approximates this functional $J$ then we can approximate the blow-up time.

In Section 3, we will apply the theorems above to three examples, where semi-discrete approximations are considered.

The first example is a generalized curvature flow, which is abbreviated to GCF. This problem is concerned with a closed convex curve which evolves with normal velocity proportional to a power of the curvature. It is well known that the solution develops singularity in finite time, see Section 3. We prove the convergence of the blow-up time by using Theorems 1.1 and 1.2.

The second example is semilinear parabolic equations of the form $u_{t}=$ $\Delta u+f(u)$, where $f(u)=u^{p}(p>1)$ or $e^{u}$. It is well known that these equations 
possess blow-up solutions. By using Theorems 1.1, 1.3 and 1.4, we prove the convergence of blow-up time. This result covers and extends the result of Abia et al $[1]$.

The final example is so-called quenching problem. We show the convergence of quenching time by using Theorem 1.2.

Finally, we would like to emphasize that the implication of our results is not limited to the numerical aspects. In fact, from our main theorems, we can see that the blow-up time depends on the initial data $u_{0}$ continuously. To see this we simply have to set $F_{h} \equiv F, B_{h} \equiv B$, and $\Omega_{h} \equiv \Omega$ in the approximate problem $\left(1.1_{\mathrm{h}}\right)$

The organization of this paper is as follows: in Section 2, we will prove Theorems 1.1, 1.2, 1.3 and 1.4. In Section 3, we apply these theorems to several examples (a generalized curvature flow, semilinear parabolic equations and quenching problem). In Section 4, we state the continuous dependence of blow-up time on the initial data.

\section{Acknowledgments}

The author would like to express his sincere gratitude to Professor Hiroshi Matano for helpful suggestions, numerous advice and continuous encouragement. The author also thanks Dr. Shigetoshi Yazaki for stimulating discussions, especially about GCF. Thanks are also due to the anonymous referee for careful reading and comments. Finally, the author would like to thank his father Teruo Ushijima for his support and advice.

\section{§2. Proof of the Main Results}

In this section we shall prove our main theorems. We note that as a direct consequence of the assumption (A2), the inequality $\liminf _{h \rightarrow 0} T_{h} \geq T$ holds. Hence what we have to prove is $\limsup _{h \rightarrow 0} T_{h} \leq T$.

Hereafter, we use the symbol $J^{\prime}$ as the derivative of $J$ with respect to $t$.

Lemma 2.1. Suppose a function $J(t)$ satisfies (I1), namely,

$$
\left\{\begin{array}{l}
J^{\prime}(t) \geq G(J), \quad t>t_{0}, \\
J\left(t_{0}\right)=J_{0}>R_{0} .
\end{array}\right.
$$

Then there exists a finite time $T$ such that $J(t)$ tends to infinity as $t \rightarrow T$ and the following estimate holds: 


$$
T \leq t_{0}+\int_{J_{0}}^{\infty} \frac{d s}{G(s)}
$$

Proof. We have $F(J(t))>0$ for $t \geq t_{0}$ since $J(t)>J\left(t_{0}\right)>R_{0}$. Hence $J^{\prime}(t) / G(J(t)) \geq 1$ holds. Integrating both sides from $t_{0}$ to $t$, we obtain

$$
t \leq t_{0}+\int_{J_{0}}^{J(t)} \frac{d s}{G(s)} .
$$

The right hand side is bounded from above. This implies the claim.

Lemma 2.2. Suppose a function $J$ satisfies (I2), namely,

$$
J^{\prime \prime}(t) \geq-c J^{\prime}(t), \quad t>t_{0} .
$$

Then we have

$$
\begin{gathered}
J(t) \geq J\left(t_{0}\right)+\frac{1-e^{-c\left(t-t_{0}\right)}}{c} J^{\prime}\left(t_{0}\right) \quad \text { if } c>0, \\
J(t) \geq J\left(t_{0}\right)+\left(t-t_{0}\right) J^{\prime}\left(t_{0}\right) \quad \text { if } c=0 .
\end{gathered}
$$

Moreover, if $J^{\prime}\left(t_{0}\right)>0$ and $J\left(t_{0}\right)<M<J\left(t_{0}\right)+\frac{J^{\prime}\left(t_{0}\right)}{c}$ then $J(t)$ reaches $M$ before $t$ becomes $t_{c}$, where we set

$$
\begin{aligned}
& t_{c}=t_{0}-\frac{1}{c} \log \left(1-\frac{c\left(M-J\left(t_{0}\right)\right)}{J^{\prime}\left(t_{0}\right)}\right) \quad \text { if } c>0, \\
& t_{c}=t_{0}+\frac{M-J\left(t_{0}\right)}{J^{\prime}\left(t_{0}\right)} \quad \text { if } c=0 .
\end{aligned}
$$

Proof. From the inequality we obtain $\left(e^{c t} J^{\prime}(t)\right)^{\prime} \geq 0$. Integrating this inequality twice, we obtain (2.1) and (2.2).

Hereafter $J(t)$ will stand for $J(u(\cdot, t))$.

Proof of Theorem 1.1.

Step $1\left(\right.$ Blow-up of $\left.u^{h}\right)$. Since $J(t)$ tends to infinity as $t \rightarrow T$, there exists $t_{0}$ such that $0<t_{0}<T$ and $J\left(t_{0}\right) \geq 2 R_{0}$. Because of the assumption (A2), there exists $h_{0}$ such that

$$
\left|J\left(t_{0}\right)-J_{h}\left(t_{0}\right)\right|<R_{0}, \quad \text { for any } h \leq h_{0} .
$$


Hence we have

$$
J_{h}\left(t_{0}\right) \geq J\left(t_{0}\right)-R_{0} \geq R_{0}
$$

for $h \leq h_{0}$. Since $J_{h}$ satisfies (I1), for $h \leq h_{0}$ the approximate solutions $u^{h}$ blow up at $T_{h}$.

Step 2 (Convergence of blow-up time). For any $\epsilon>0$ there exists a number $R \geq R_{0}$ such that

$$
\int_{R}^{\infty} \frac{d s}{G(s)}<\epsilon
$$

For this $R$ there exist $t_{1}$ and $h_{1}$ such that

$$
J_{h}\left(t_{1}\right) \geq J\left(t_{1}\right)-R \geq R
$$

for $h \leq h_{1}$. By virtue of Lemma 2.1, the blow-up time $T_{h}$ of approximate solutions satisfies

$$
T_{h} \leq t_{1}+\int_{J_{h}\left(t_{1}\right)}^{\infty} \frac{d s}{G(s)} \leq T+\epsilon
$$

for any $h \leq h_{1}$. Consequently we have $\limsup _{h \rightarrow 0} T_{h} \leq T$.

Proof of Theorem 1.2. We will consider only the case where $c>0$, since the case where $c=0$ can be treated similarly.

Step 1. We claim that for any $\delta \geq 0$ there exists $h_{1}>0$ such that for each $h \leq h_{1}$ there exists $\bar{t}_{1}=\bar{t}_{1}(h)$ which satisfies

$$
J_{h}^{\prime}\left(\bar{t}_{1}\right) \geq \delta
$$

In fact, for any $\delta \geq 0$, there exists a time $t_{1}<T$ such that for any $t \geq t_{1}$

$$
J^{\prime}(t) \geq 2 \delta \geq 0
$$

since $J^{\prime}(t)$ tends to infinity. Without loss of generality we can assume that $T-t_{1}<1$. Now set $K_{1}=\left[t_{1},\left(t_{1}+T\right) / 2\right]$ and denote by $\left|K_{1}\right|$ the length of the interval $K_{1}$. For $\nu \leq \frac{\left|K_{1}\right| \delta}{2}$, there exists $h_{1}$ such that for any $h \leq h_{1}$

$$
\sup _{t \in K_{1}}\left|J_{h}(t)-J(t)\right| \leq \nu .
$$

By virtue of the mean value theorem, we see that for any $h \leq h_{1}$ there exist $\bar{t}_{1}=\bar{t}_{1}(h) \in K_{1}$ and $t_{1}^{\prime} \in K_{1}$ such that

$$
\left|J_{h}^{\prime}\left(\bar{t}_{1}\right)-J^{\prime}\left(t_{1}^{\prime}\right)\right| \leq \frac{2 \nu}{\left|K_{1}\right|} \leq \delta .
$$


Hence we have the claim.

Step 2(Blow-up of $\left.u^{h}\right)$. By the result of the previous step there exists $h_{1}$ such that $J_{h}^{\prime}(t)>0$ for any $h \leq h_{1}$ and some $t$. Because of the condition (A1') we can prove the blow-up of $u^{h}$ by Lemma 2.2 .

Step 3(Convergence of blow-up time). Making use of Lemma 2.2 and the first step, we have

$$
J_{h}(t) \geq J_{h}\left(\bar{t}_{1}\right)+\frac{1-e^{-c\left(t-\bar{t}_{1}\right)}}{c} J_{h}^{\prime}\left(\bar{t}_{1}\right) \geq J\left(\bar{t}_{1}\right)-\frac{\left|K_{1}\right| \delta}{2}, \quad t \geq \bar{t}_{1} .
$$

Therefore we can assume that there exists a constant $m$ and time $t_{*}$ such that

$$
J_{h}(t) \geq m \quad \text { for } t \geq t_{*}, h \leq h_{1} .
$$

For any $\epsilon>0$ and $M>m$ there exists a number $R>0$ such that

$$
0<-\frac{1}{c} \log \left(1-\frac{c(M-m)}{R}\right) \leq \epsilon .
$$

Applying the result in Step 1, we can choose a number $h_{2}$ such that for any $h \leq h_{2}$ there exists a time $\bar{t}_{2}$ such that $T>\bar{t}_{2}>t_{*}$ and $J_{h}^{\prime}\left(\bar{t}_{2}\right) \geq R$. By means of Lemma 2.2, for all $h \leq h_{2}$

$$
T_{h} \leq \bar{t}_{2}-\frac{1}{c} \log \left(1-\frac{c\left(M-J_{h}\left(\bar{t}_{2}\right)\right)}{J_{h}^{\prime}\left(\bar{t}_{2}\right)}\right) \leq T-\frac{1}{c} \log \left(1-\frac{c(M-m)}{R}\right) \leq T+\epsilon .
$$

Consequently we have $\limsup _{h \rightarrow 0} T_{h} \leq T$.

Proof of Theorem 1.3. Again we will prove only in the case where $c>0$. Using the same argument as in Step 3 of the previous proof, we can assume that there exist constants $m, h_{1}$, and $t_{1}$ such that $J_{h}(t) \geq m$ for $t \geq t_{1}$ and $h \leq h_{1}$.

For any positive $\epsilon$ there exists $R>R_{0}$ such that

$$
\int_{R}^{\infty} \frac{d s}{G(s)} \leq \frac{\epsilon}{2}
$$

We set $t_{0}=T+\frac{\epsilon}{2}$. There exists a time $t_{2}<T$ such that

$$
J^{\prime}\left(t_{2}\right) \geq \frac{2(R-m) c}{1-e^{-c\left(t_{0}-T\right)}},
$$

since $J^{\prime}(t)$ tends to infinity. By the same argument as in Step 1 in the previous proof, there exists $h_{2}$ and for any $h \leq h_{2}$ there exists $\bar{t}_{2}=\bar{t}_{2}(h)<T$ such that

$$
J_{h}^{\prime}\left(\bar{t}_{2}\right) \geq \frac{(R-m) c}{1-e^{-c\left(t_{0}-T\right)}} .
$$


By virtue of Lemma 2.2, for any $h \leq h_{2}$ we have

$$
J_{h}\left(t_{0}\right) \geq J_{h}\left(\bar{t}_{2}\right)+\frac{1-e^{-c\left(t_{0}-\bar{t}_{2}\right)}}{c} J_{h}^{\prime}\left(\bar{t}_{2}\right) \geq m+\frac{1-e^{-c\left(t_{0}-\bar{t}_{2}\right)}}{1-e^{-c\left(t_{0}-T\right)}}(R-m) \geq R .
$$

From Lemma 2.1, we can conclude that the approximate solution must blow up in finite time $T_{h}$ and for $h \leq h_{2}$

$$
T_{h} \leq t_{0}+\int_{R}^{\infty} \frac{d s}{G(s)} \leq T+\epsilon
$$

Hence we have $\limsup _{h \rightarrow 0} T_{h} \leq T$.

Proof of Theorem 1.4. Since we assume that

$$
J_{h}^{\prime}(t) \geq H_{h}(t), \quad H_{h}^{\prime}(t) \geq 0
$$

and

$$
J_{h}^{\prime}(t) \geq G\left(J_{h}(t)\right)
$$

we have the following estimates

$$
t_{1}-t_{0} \leq \frac{J_{h}\left(t_{1}\right)}{H_{h}\left(t_{0}\right)}
$$

and

$$
t_{1}-t_{0} \leq \int_{J_{h}\left(t_{0}\right)}^{J_{h}\left(t_{1}\right)} \frac{d s}{G(s)} .
$$

For any $\varepsilon>0$ there exists $R>0$ such that

$$
\int_{R}^{\infty} \frac{d s}{G(s)}<\varepsilon
$$

By the assumption $\left(\mathrm{b}^{\prime}\right)$ and $\left(\mathrm{A} 2^{\prime}\right)$, there exists $\delta>0$ and $h_{0}>0$ such that for any $h \leq h_{0}$

$$
H_{h}(T-\delta) \geq \frac{2 R}{\varepsilon}
$$

Let us assume that $T_{h} \geq T+\varepsilon / 2$ for an $h \leq h_{0}$. By the inequality (2.3) we have

$$
J_{h}(T+\varepsilon / 2) \geq(\varepsilon / 2+\delta) H_{h}(T-\delta) \geq R
$$


By the inequality (2.4) we obtain

$$
T_{h}-(T+\varepsilon / 2) \leq \int_{J_{h}(T+\varepsilon / 2)}^{J_{h}\left(T_{h}\right)} \frac{d s}{G(s)} \leq \int_{R}^{\infty} \frac{d s}{G(s)} \leq \varepsilon / 2 .
$$

Hence we have $T_{h} \leq T+\varepsilon$.

\section{$\S 3 . \quad$ Examples}

In this section, we present three examples. The first example is a generalized curvature flow. The second is semilinear parabolic partial differential equations of the form $u_{t}=\Delta u+f(u)$. And the last one is a quenching problem. We apply Theorems 1.1 and 1.2 to the first one, Theorems 1.2, 1.3 and 1.4 to the second one, and Theorem 1.3 to the third one.

\section{§3.1. Generalized curvature flow}

In this subsection, we apply our theorems to a generalized curvature flow problem. We first explain this problem briefly. We then introduce an approximate problem which is based on so-called crystalline approximation of smooth curves. For this approximate problem, we show the convergence of blow-up time.

\section{Generalized Curvature Flow}

The problem is to describe the motion of closed immersed curve $\mathbf{C}(t)=$ $\left\{X(t) \in \mathbb{R}^{2}\right\}$ which is evolved by the law

$$
V=\frac{1}{\alpha} K^{\alpha}
$$

We assume that the curvature $K$ is positive everywhere. The symbols $V$ and $\alpha$ denote the outward normal velocity and nonzero parameter, respectively.

When $\alpha=1$, the problem (3.1) is called the problem of curvature flow. There are many papers which study this problem (see [7], [19], [24], and references therein). When $\alpha=1 / 3$, the problem (3.1) is invariant under affine transformation [38] and attracts many researchers in recent years (see [6] and references therein). Moreover, the evolution law (3.1) with $\alpha=1 / 3$ is used for a model equation for image processing [2]. We call the problem (3.1) generalized curvature flow problem (GCF in short).

Since the curvature $K$ is positive everywhere, we can parameterize the curve by the normal direction $\theta$. Then GCF is described by the following 
initial-boundary value problem for a quasilinear parabolic partial differential equation:

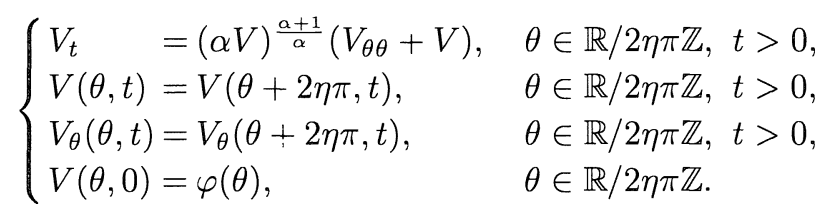

Here $\eta$ denotes the rotation number of the curve. We assume that the initial data $\varphi$ is a smooth function which satisfies $\int_{0}^{2 \eta \pi} \frac{e^{2 \theta}}{\varphi^{1 / \alpha}} d \theta=0$ and $\alpha \varphi>0$. As for the derivation of this equation, see for instance [5].

We explain several properties of GCF (see [3], [4], [7] for more precise information). Firstly the problem (3.2) possesses a unique smooth local solution which does not change sign. Secondly, GCF is a curve-shortening and areadecreasing motion for $\alpha>0$, and is a curve-lengthening and area-increasing motion for $\alpha<0$. Namely, $\frac{d}{d t} L \leq 0$ and $\frac{d}{d t} A \leq 0$ hold true for $t>0$ if $\alpha>0$ : the opposite inequalities hold true if $\alpha<0$. Here $L$ and $A$ denote the length of the curve and the area enclosed by the curve, respectively. Thirdly, for $\alpha>0$ and $\alpha<-1$ all solutions of GCF develop singularity in finite time, say $T$ : at $t=T, L^{\infty}$ norm of the solution $V$ diverges. This fact can be proved by the comparison lemma. Finally, in the blow-up case, regional blow-up occurs (see Proposition 3.1 bellow).

Let us introduce the notion of the blow-up set. The set

$S(V)=\left\{x \in \Omega \mid \exists x_{n}, \exists t_{n}\right.$ s.t. $\left.\lim _{n \rightarrow \infty}\left|V\left(t_{n}, x_{n}\right)\right|=\infty, \lim _{n \rightarrow \infty} x_{n}=x, \lim _{n \rightarrow \infty} t_{n}=T\right\}$

is called the blow-up set of the solution $V$. When the measure of the blow-up set is positive, we call it regional blow-up. The following proposition holds true (see [4], [20] for more precise information).

Proposition 3.1. For any blow-up solution $V$, the Lebesgue measure of the blow-up set $S(V)$ is greater than or equal to $\pi$.

Proof. We assume that $\alpha>0$. The case where $\alpha<-1$ can be treated similarly. Suppose the contrary, then there exist intervals $(a, b),\left(a_{0}, b_{0}\right)$ and a finite constant $M$ such that $b-a<\pi, S(V) \subset\left(a_{0}, b_{0}\right) \subset(a, b), V\left(t, a_{0}\right) \leq M$, and $V\left(t, b_{0}\right) \leq M$. For a constant $A>0$, we define $\varphi(\theta)=A \cos \frac{\pi}{b-a}\left(x-\frac{a+b}{2}\right)$. Since $\cos \frac{\pi}{b-a}\left(\frac{b_{0}-a_{0}}{2}\right)>0$, both $\varphi\left(a_{0}\right)$ and $\varphi\left(b_{0}\right)$ are greater than or equal to $M$ for sufficiently large $A$. Moreover, $\varphi$ satisfies $\varphi^{\prime \prime}+\varphi=\left(1-\left(\frac{\pi}{b-a}\right)^{2}\right) \varphi<0$, hence $\varphi_{t}=0>(\alpha V)^{\frac{\alpha+1}{\alpha}}\left(\varphi_{\theta \theta}+\varphi\right)$. On the other hand $V$ is a solution of (3.2). 
By virtue of the maximum principle of parabolic type, we have $V(t, \theta) \leq \varphi(\theta)$ for $\theta$ in $\left(a_{0}, b_{0}\right)$. It contradicts the blow-up of $V$.

\section{Approximate Problem}

Let us consider an approximate problem which is given by the system of ordinary differential equations for $v_{\jmath}^{n}$ :

$$
\begin{cases}\frac{d}{d t} v_{\jmath}^{h}=\left(\alpha v_{\jmath}^{h}\right)^{\frac{a+1}{a}}\left(\Delta_{\theta} v^{h}+v^{h}\right)_{\jmath}, & 0 \leq j \leq n, \\ v_{\jmath}^{h}(t)=v_{\jmath+n}^{h}(t), & 0 \leq j \leq n, \\ v_{\jmath}^{h}(0)=\varphi_{\jmath}, & 0 \leq j \leq n,\end{cases}
$$

where $\Delta \theta=2 \eta \pi / n$ and

$$
\left(\Delta_{\theta} v\right)_{\jmath}=\frac{v_{\jmath+1}-2 v_{\jmath}+v_{\jmath-1}}{2(1-\cos \Delta \theta)} .
$$

This approximate problem is derived by the so-called crystalline approximation (see [40], [41]).

Several authors studied numerical methods for problem (3.1) and related problems. Kimura $[28,29]$ constructed a numerical scheme for (3.1) with $\alpha=1$ and proved its convergence. Mikula [33] studied a numerical scheme for equations which are related to (3.1) and (3.2). Recently Mikula and Ševčovič [34] developed an effective numerical scheme based on so-called intrinsic heat equation. Ishiwata and Tsutsumi [26] investigated numerical treatment for equation (3.2) under Dirichlet boundary condition. Using crystalline approximation, we constructed a numerical scheme for this problem and proved the convergence of the scheme [40]. Our scheme enjoys the discrete versions of the properties of GCF.

We note that the solution of (3.3) does not change sign. This can be proved by the discrete comparison lemma. We assume $\alpha>0$ or $\alpha<-1$, namely $V$ blows up in finite time $T$.

We define functionals $J$ and $J_{h}$ as follows:

$$
\left.J_{[}^{[} V\right](t)=-\operatorname{sgn}(\alpha) \int_{0}^{2 \eta \pi}(\alpha V)^{-1 / \alpha} d \theta
$$

and

$$
J_{h}\left[v^{h}\right](t)=-\operatorname{sgn}(\alpha) \sum_{\jmath=0}^{n-1}\left(\alpha v_{\jmath}^{h}\right)^{-1 / \alpha} \Delta \theta .
$$

Let us check that the functionals $J$ and $J_{h}$ satisfy the assumptions of Theorem 1.2 and Theorem 1.1 for $\alpha>0$ and $\alpha<-1$, respectively. 
Firstly we can prove that the convergence between the solution $v^{h}$ of the problem (3.3) and the solution $V$ of the problem (3.2).

Proposition 3.2. $T$ denotes the blow-up time of the solution $V$ to (3.2). We assume $\max _{0 \leq j \leq n}\left|\varphi(j \Delta \theta)-\varphi_{\jmath}\right|=o(1)$ as $\Delta \theta$ tends to zero. For any $\epsilon$ we have

$$
\lim _{n \rightarrow \infty} \max _{t \in[0, T-\epsilon], 0 \leq \jmath \leq n}\left|V(j \Delta \theta, t)-v_{\jmath}^{h}(t)\right|=0 .
$$

Proof. This result can be obtained by a discrete analogue of super and subsolution method (see appendix).

We set $v_{\jmath}^{ \pm}(t)=V(j \Delta \theta, t) \pm A e^{B t} m(\Delta \theta)$, where $m$ is a function of $\Delta \theta$ such that $m(\Delta \theta)=o(1)$ and $\max _{0 \leq j \leq n}\left|\varphi(j \Delta \theta)-\varphi_{\jmath}\right|=o(1)$ as $\Delta \theta \rightarrow 0$. For suitable $A, B$, and sufficiently large $n$, we can check that $v_{\jmath}^{+}(t)\left(v_{\jmath}^{-}(t)\right)$ is a supersolution (subsolution, respectively) for the equation (3.3). Therefore we can prove that there exists a constant $C_{\epsilon}$ such that for sufficiently large $n$

$$
\max _{0 \leq t \leq T-\epsilon}\left|V(t, j \Delta \theta)-v_{\jmath}^{h}(t)\right| \leq C_{\epsilon} m(\Delta \theta)
$$

by the comparison lemma (Theorem A.1 below).

Using this convergence result we can easily check that the condition (A2) holds.

Secondly, we can prove the following result. The continuous part of the proposition was first proved by Gage [20] for the case of the Dirichlet boundary condition. By this result, we can check that the inequality (I2) holds for $\alpha>0$.

Proposition 3.3. We assume that $\alpha>0$. There exists a positive constant $c$ which depends only on the initial data such that

$$
V_{t}(t, \theta) \geq-c V(t, \theta) \quad \text { and } \quad \frac{d}{d t} v_{\jmath}^{h}(t) \geq-c v_{\jmath}^{h}(t, \theta) .
$$

Proof. We set $c=\max \left\{-\min _{\theta} \alpha V_{0}(\theta)^{\frac{1}{\alpha}}\left(\left(V_{0}\right)_{\theta \theta}(\theta)+V_{0}(\theta)\right), 0\right\}(\geq 0)$ and $w(t, \theta)=V_{t}(t, \theta)+c V(t, \theta)$. By the definition of $c$, we have $w(0, \theta) \geq 0$.

One can easily check that $w$ satisfies

$$
\begin{aligned}
w_{t} & =\alpha V^{\frac{\alpha+1}{\alpha}} w_{\theta \theta}+\left(\alpha V^{\frac{\alpha+1}{\alpha}}-\frac{(3 \alpha+2) c}{\alpha}\right) w+\frac{(\alpha+1) w^{2}}{\alpha V}+\frac{(2 \alpha+1) c^{2} V}{\alpha} \\
& \geq \alpha V^{\frac{\alpha+1}{\alpha}} w_{\theta \theta}+\left(\alpha V^{\frac{\alpha+1}{\alpha}}-\frac{(3 \alpha+2) c}{\alpha}\right) w,
\end{aligned}
$$


since $V>0$ and $\alpha>0$.

For a positive number $k$, we set $W(t, \theta)=e^{-k t} w(t, \theta)$. Then $W$ satisfies

$$
W_{t} \geq \alpha V^{\frac{\alpha+1}{\alpha}} W_{\theta \theta}+\left(\alpha V^{\frac{\alpha+1}{\alpha}}-c \frac{3 \alpha+2}{\alpha}-k\right) W .
$$

We fix a time $t_{0}$ such that $V$ is bounded for $t \in\left[0, t_{0}\right]$, then there exists a positive number $k$ such that $\left(\alpha V^{\frac{\alpha+1}{\alpha}}-c \frac{3 \alpha+2}{\alpha}-k\right)<0$. From the maximum principle, we obtain $W(t, \theta) \geq 0$ for any $t \in\left[0, t_{0}\right]$. Hence we have $w(t, \theta) \geq 0$ for any $t \in\left[0, t_{0}\right]$. It means that $V_{t} \geq-c V$ holds as long as $V$ is bounded.

By an analogous argument, we can prove the latter part of the proposition.

Let us assume that $\alpha>0$. By means of Proposition 3.1, $\frac{d}{d t} J[V](t)=$ $\frac{1}{\alpha} \int_{0}^{2 \eta \pi} V d \theta$ tends to infinity as $t \rightarrow T$. From Proposition 3.3 we can check that the inequality (I2) holds:

$$
\frac{d^{2}}{d t^{2}} J_{h}\left[v^{h}\right](t)=\frac{1}{\alpha} \sum_{\jmath=0}^{n-1} \frac{d}{d t} v_{\jmath}^{h}(t) \Delta \theta \geq-c \frac{d}{d t} J_{h}\left[v^{h}\right](t) .
$$

Functionals $J$ and $J_{h}$ are bounded from above, since $V$ and $v^{h}$ are bounded from below. Finally Proposition 3.2 ensures (A2) (see [40]). Hence we can obtain the convergence of blow-up time by using Theorem 1.2.

Let us now assume that $\alpha<-1$. We can check that $J$ and $J_{h}$ satisfies (A1) and (A2). Since $\alpha<-1, J$ tends to infinity as $t \rightarrow T$, because of Proposition 3.1. Proposition 3.2 also ensures (A2). By Jensen's inequality, there exists a constant $c=c(\eta)$ such that

$$
\frac{d}{d t} J_{h} \geq-\frac{c}{\alpha} J_{h}^{-\alpha}
$$

So the inequality (I1) holds with $G(s)=-\frac{c}{\alpha} s^{-\alpha}$. Hence the convergence of blow-up time holds true by Theorem 1.1.

We have thus proved the following.

Theorem 3.1. We assume $\alpha>0$ or $\alpha<-1$. T denotes the blow-up time of $V$. We assume $\max _{0 \leq j \leq n}\left|\varphi_{j}-\varphi(j \Delta \theta)\right|=o(1)$ as $n$ tends to infinity. Then for sufficiently large $n$, the solution $v^{h}(t)$ of the problem (3.3) blows up in finite time $T_{n}$. Moreover, we have

$$
\lim _{n \rightarrow \infty} T_{n}=T
$$




\section{§3.2. Semilinear parabolic equations}

In this subsection, we shall treat semilinear parabolic equation:

$$
\begin{cases}u_{t}(x, t)=\Delta u+f(u), & x \in \Omega, t>0 \\ u(x, 0)=u_{0}(x), & x \in \Omega, t=0 \\ u(x, t)=0, & x \in \partial \Omega, t>0 .\end{cases}
$$

Here $\Omega$ is a bounded domain in $\mathbb{R}^{N}$. We assume that initial data $u_{0}$ is a nonnegative smooth function and $u_{0}(x)=0(x \in \partial \Omega)$. We consider the case where $f(u)=u^{p}(p>1)$ or $e^{u}$. The former nonlinearity is called of Fujita type and the latter arises from combustion theory (see [9], for instance).

It is well known that for sufficiently large initial data, solution of this problem do blow-up in finite time. See, for instance, [18] and [31].

The blow-up solutions of this problem were extensively studied and now rather precise information near blow-up time is clarified (see [37], [42], and references therein).

As for numerical approach, some excellent schemes were invented ([10][12]). Some authors studied numerical blow-up time and its convergence. Nakagawa [35] studied a finite difference scheme for $u_{t}=u_{x x}+u^{2}$ and proved the convergence of blow-up time. Nakagawa and Ushijima [36] studied a finite element full-discrete scheme for the semilinear heat equation of blow-up type based on a blow-up criterion due to Kaplan and Fujita. Chen [13] also studied a finite difference scheme for $u_{t}=u_{x x}+u^{p}, p>1$ and proved the convergence of blow-up time. They all assumed that the $L^{q}$ ( $q=1$ or 2 ) norm of the solution tends to infinity as $t$ approaches the blow-up time $T$. However, it does not always hold (see Remark 3.1.). Recently, Abia, López-Marcos and Martínez [1] considered one dimensional semi-discrete problem for (3.4) with more general nonlinear term $f$. They proved the convergence of blow-up time, on the condition that $L^{\infty}$ norm of solution blows up.

In this subsection, we prove the convergence of blow-up time for $\mathrm{N}$ dimensional semi-discrete problem, using our general theory, under the assumption that the $L^{\infty}$ norm blows up. Our result contains the result of [1] as a special case (see Remark 3.3).

\section{Blow-up Problem of Fujita Type}

First we treat the case where $f=u^{p}(p>1)$. We define the energy $I$ by

$$
I[u](t)=\frac{1}{2} \int_{\Omega}|\nabla u|^{2} d x-\frac{1}{p+1} \int_{\Omega} u^{p+1} d x .
$$


For any solution $u$, this $I$ is monotone non-increasing function of $t$. In fact, one can easily check that $\frac{d}{d t} I\left[u_{j}^{\top}(t)=-\int_{\Omega} u_{t}^{2} d x \leq 0\right.$. Let us define the functional $J$ as follows:

$$
J[u](t)=\int_{\Omega} u^{2}(x, t) d x .
$$

For this $J$ we can verify that

$$
\begin{aligned}
\frac{d}{d t} J[u] & =2 \int_{\Omega} u u_{t} d x \\
& =-4 I[u]+\frac{2(p-1)}{p+1} \int_{\Omega} u^{p+1} d x \\
& \geq-4 I\left[u_{0}\right]+c(\Omega)\left(J\left[u_{]}^{]}\right)^{\frac{p+1}{2}} .\right.
\end{aligned}
$$

Here we have used Jensen's inequality.

Proposition 3.4. We assume that the $L^{\infty}$ norm of the solution $u$ to the problem (3.4) tends to infinity as $t$ tends to $T$. We also assume $q>\frac{N(p-1)}{2}$ then we have

$$
\lim _{t \rightarrow T}\|u(\cdot, t)\|_{q}=\infty .
$$

Here, $\|u\|_{q}$ denotes the $L^{q}$ norm of solution $u$.

Remark 3.1. This fact is well known for the specialists. For proof of the proposition, we refer Giga [21] and Friedman and McLeod [17]. Friedman and McLeod [17] also show that if $\Omega$ is convex and $q<\frac{N(p-1)}{2}$ then there exists initial data such that

$$
\limsup _{t \rightarrow T}\|u(\cdot, t)\|_{q}<\infty .
$$

Accordingly we can not always expect $J$ tends to infinity as $t$ tends to the blow-up time.

Proposition 3.5. Assume $1<p<\frac{N+2}{N-2}$, then $-I(t)$ tends to infinity as $t$ approaches $T$.

This result was proved by Giga [22].

Let us introduce an approximate problem. We set $\Omega=(0,1)^{N}$ for simplicity. We approximate the problem by the semi-discrete problem:

$$
\left\{\begin{array}{lll}
\dot{u}_{K}=\left(\Delta_{h} u\right)_{K}+f\left(u_{K}\right), & & K \in \mathcal{K}, t>0, \\
u_{K}(t)=0, & & K \in \mathcal{K}_{0}, t>0 \\
u_{K}(0)=u_{0 K}, & & K \in \mathcal{K} .
\end{array}\right.
$$


Here we have introduced a square grid with mesh size $h=1 / n$ in domain $\Omega$, the function $u_{K}(t)$ is defined on this grid. The set of indices $K=\left(j_{1}, \ldots, j_{N}\right)$ is denoted by $\mathcal{K}$ and the set of indices $K$ corresponding to the boundary is denoted by $\mathcal{K}_{0}$. Namely,

$$
\mathcal{K}=\left\{\left(j_{1}, \ldots, j_{N}\right): 0 \leq j_{1}, \ldots, j_{N} \leq n\right\}
$$

and

$$
\mathcal{K}_{0}=\left\{\left(j_{1}, \ldots, j_{N}\right) \in \mathcal{K}: j_{\imath}=0 \text { or } n\right\}
$$

We set $K h=\left(j_{1} h, j_{2} h, \ldots, j_{N} h\right)$. We define a discrete Laplace operator $\Delta_{h}$ by

$$
\left(\Delta_{h} u\right)_{K}=\sum_{\imath=1}^{N}\left(\Delta_{h_{\imath}} u\right)_{\jmath_{1}, \ldots, \jmath_{N}}
$$

where we set

$$
\left(\Delta_{h_{\imath}} u\right)_{\jmath_{1}, \ldots, \jmath_{N}}=\frac{u_{\jmath_{1}, \ldots, \jmath_{2}-1, \ldots, \jmath_{N}}-2 u_{\jmath_{1}, \ldots, \jmath_{2}, \ldots, \jmath_{N}}+u_{\jmath_{1}, \ldots, \jmath_{2}+1, \ldots, \jmath_{N}}}{h^{2}} .
$$

The dot in (3.9) denotes derivative with respect to $t$.

For this approximate problem, we can prove the convergence result by a discrete analogue of super and subsolution method.

Proposition 3.6. We assume the solution $u$ of (3.4) blows up at finite time $T$ and $\max _{K \in \mathcal{K}}\left|u_{0}(K h)-u_{0 K}^{n}\right|=o(1)$ as $h \rightarrow 0$. For any $\epsilon$ we have

$$
\lim _{n \rightarrow \infty} \max _{t \in[0, T-\epsilon], K \in \mathcal{K}}\left|u(K h, t)-u_{K}^{n}(t)\right|=0 .
$$

Proof. We set $u_{K}^{ \pm}(t)=u(K h, t) \pm A e^{B t} \varphi(K h) m(h)$, where $m(h)$ is a function of $h$ such that $m(h)=o(1)$ as $h$ tends to zero and $\max _{K \in \mathcal{K}} \mid u_{0}(K h)-$ $u_{0 K}^{n} \mid \leq m(h), \varphi(x)$ is the first eigenfunction of $-\Delta$ with homogeneous Dirichlet boundary condition. For suitable $A, B$, and sufficiently small $h$, we can prove that $u^{+}$and $u^{-}$are super and subsolution, respectively. From the comparison lemma, we obtain the assertion.

Let us define functionals $I_{n}$ and $J_{n}$ as follows:

$$
I_{n}\left[u^{n}\right](t)=\frac{1}{2} \sum_{K \in \mathcal{K}} \sum_{\imath=1}^{N}\left(D_{h_{\imath}} u^{n}\right)_{K}^{2} h^{N}-\frac{1}{p+1} \sum_{K \in \mathcal{K}}\left(u_{K}^{n}\right)^{p+1} h^{N},
$$


and

$$
J_{n}\left[u^{n}\right](t)=\sum_{K \in \mathcal{K}}\left(u_{K}^{n}\right)^{2} h^{N}
$$

Here we set

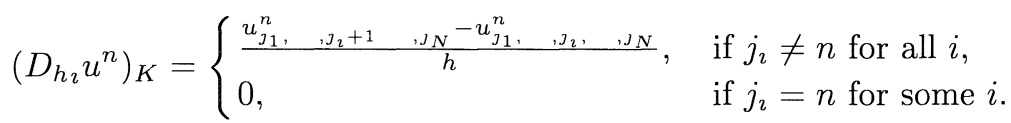

Because of the definition of difference operators above, the formula of summation by parts holds. Thus we can prove the semi-discrete analogue of (3.7) and (3.8). Namely, we have

$$
\begin{aligned}
\frac{d}{d t} J_{n}\left[u^{n}\right] & =-4 I_{n}\left[u^{n}\right]+\frac{2(p-1)}{p+1} \sum_{K \in \mathcal{K}}\left(u_{K}^{n}\right)^{p+1} h^{N} \\
& \geq-4 I_{n}\left[u_{0}^{n}\right]+c(\Omega)\left(J_{n}\left[u^{n}\right]\right)^{\frac{p+1}{2}}
\end{aligned}
$$

since $\dot{I}_{n}=-\sum_{K \in \mathcal{K}} \dot{u}_{K}^{2} h^{N} \leq 0$.

Now we state our result.

Theorem 3.2. We assume that the solution of (3.4) blows up in finite time $T$ and $\max _{K}\left|u_{0}(K h)-u_{0 K}^{n}\right|=o(1)$. Assume one of the following.

1. $1<p<\frac{N+2}{N-2}$ or $-I(t)$ tends to infinity as $t$ tends to $T$.

2. $1<p<1+4 / N$ or $J[u](t)$ tends to infinity as $t$ tends to $T$.

Then for sufficiently large $n$ the approximate solution blows up in finite time $T_{n}$ and we have $\lim _{n \rightarrow \infty} T_{n}=T$.

Proof. The first part of the theorem is proved by Theorem 1.4 and the second part is proved by Theorem 1.1.

By means of Proposition 3.2 we can verify that the assumption (A2) holds for $J$ and $J_{n}$.

Proof of 1. By Proposition 3.5, $-I(t)$ tends to infinity as $t \rightarrow T$. Set $H(t)=-2 I(t)$ and $H_{n}(t)=-2 I_{n}(t)$ then the condition (A2') holds. Since we have the inequality (3.11) we obtain the conclusion by Theorem 1.4.

Proof of 2. From Proposition 3.4, the condition $1<p<1+\frac{4}{N}$ ensures that $J[u](t)$ tends to infinity as $t$ tends to $T$. Since the inequality (3.11) holds, the assumption (A1) is satisfied. By virtue of Theorem 1.1, we obtain the result. 
Remark 3.2. Our theorem is valid for more general nonlinearity $f$.

Firstly if we assume

$$
0<\lim _{z \rightarrow \infty} \frac{f(z)}{z^{p}}<\infty
$$

for $1<p<\frac{N+2}{N-2}$ then $-I(t)$ tends to infinity as $t \rightarrow T([22])$. Here, for such nonlinearity $f$ we modify the energy $I$ as follows:

$$
\begin{gathered}
I(t)=\frac{1}{2} \int_{\Omega}|\nabla u|^{2} d x-\int_{\Omega} F(u) d x, \\
F(u)=\int_{0}^{u} f(s) d s .
\end{gathered}
$$

Secondly if we assume that there exists a constant $L$ such that

$$
|f(y)-f(z)| \leq L|y-z|\left(|y|^{p-1}+|z|^{p-1}\right), \quad f(0)=0,
$$

for $1<p<1+4 / N$ then $\|u\|_{2} \rightarrow \infty$ as $t \rightarrow T([21])$.

Thirdly if we assume that there exists positive constants $c_{1}$ and $c_{2}$ such that

$$
u f(u)-2 F(u) \geq c_{1}|u|^{p+1}-c_{2},
$$

then we can do the calculation that are analogous to (3.7) and (3.8). These analogues give us the finite time blow-up and the convergence of blow-up time.

Hence, the first part of Theorem 3.2 holds with $f$ which satisfies (3.12) and (3.14), and the second holds with $f$ which satisfies (3.13) and (3.14).

Remark 3.3. The result of Abia, López-Marcos and Martínez [1] required strong assumption on initial data: there exists a positive constant $a$ such that

$$
\left(\Delta_{h} u_{0}^{n}\right)_{K}+f\left(u_{0 K}^{n}\right) \geq a f\left(u_{0 K}^{n}\right) .
$$

Also they considered only the one-dimensional problem.

The Case where $f(u)=e^{u}$

We employ the same approximate problem as the previous one. For the case where $f(u)=e^{u}$, Propositions 3.6 and 3.7 remain valid.

Let us construct the functionals $J$ and $J_{n}$ which satisfy our assumptions. Let $\varphi(x)$ and $\lambda$ denote a first eigenfunction of $-\Delta$ under the Dirichlet boundary 
condition and corresponding eigenvalue, respectively. We define the functionals $J$ and $J_{n}$ by

$$
J[u](t)=\int_{\Omega} u \varphi d x
$$

and

$$
J_{n}\left[u^{n}\right](t)=\sum_{K \in \mathcal{K}} u_{K}^{n} \varphi(K h) h^{N}
$$

For these functionals, we have the following: there exist positive constants $c_{1}$ and $c_{2}$ such that

$$
J^{\prime}(t)=-\lambda J(t)+\int_{\Omega} e^{u} \varphi d x \geq-\lambda J+c_{1} e^{c_{2} J},
$$

and

$$
J_{n}^{\prime}(t) \geq-\lambda_{n} J_{n}(t)+c_{1} e^{c_{2} J_{n}}
$$

where $\lambda_{n}$ is the first eigenvalue of $-\Delta_{h}$. We note that $\lim _{n \rightarrow \infty} \lambda_{n}=\lambda$.

Here if we assume that $\lim _{t \rightarrow T} J[u](t)=\infty$, then there exist a positive constant $c_{3}$ and $t_{0}<T$ such that $J_{n}^{\prime}(t) \geq c_{3} e^{c_{2} J_{n}}$ for all $t \geq t_{0}$. Thus we can obtain the convergence of blow-up time by Theorem 1.1. However, we could not find any sufficient condition which ensures $\lim _{t \rightarrow T} J\left[u_{!}(t)=\infty\right.$ (see Remark 3.4). So in the following we will restrict ourselves to a narrow class of initial data and apply Theorem 1.3, in order to obtain the convergence of blow-up time.

We note that if the initial data is a subsolution then the solution is monotone non-decreasing function with respect to $t$. This fact is easily proved by using the comparison lemma.

Proposition 3.7. We assume that

$$
\left(\Delta_{h} u_{0}^{n}\right)_{K}+f\left(u_{0 K}^{n}\right) \geq 0 .
$$

Then we have

$$
\dot{u}_{K}^{n}(t) \geq 0 \text {. }
$$

Hence if we assume (F1) then we have

$$
J_{n}^{\prime \prime}(t) \geq-\lambda_{n} J_{n}^{\prime}(t) .
$$


If we further assume

$$
\lim _{t \rightarrow T} \int_{\Omega} e^{u} \varphi d x=\infty
$$

then we obtain the convergence of blow-up time by Theorem 1.3. Therefore we have the following:

Theorem 3.3. We assume that the solution of (3.4) blows up in finite time $T$ and $\max _{K \in \mathcal{K}}\left|u_{0}(K h)-u_{0 K}^{n}\right|=o(1)$. Assume one of the following holds.

1. $J[u](t)$ tends to infinity as $t$ tends to $T$.

2. (F1) and (F2) hold.

Then for sufficiently large $n$ the approximate solution blows up in finite time $T_{n}$ and we have $\lim _{n \rightarrow \infty} T_{n}=T$.

Remark 3.4. In the case where the domain is a ball and the initial data is radially symmetric, the assumption $\lim _{t \rightarrow T} J[u](t)=\infty$ does not hold. See Friedman and McLeod [17].

Remark 3.5. The assumption (F2) holds when $N=1$. In fact, we can prove it by the same argument in Friedman and McLeod [17].

It is known that for any $b \in \Omega$ there exists a blow-up solution $u$ which blows up at $(b, T) \in \mathbb{R}^{N} \times(0, \infty)$ and whose final time blow-up profile satisfies

$$
u(x, T)=-2 \ln |x-b|+\ln |\ln | x-b||+\ln 8+o(|x-b|),
$$

provided $\Omega$ is a convex domain ([8], [11]). Moreover, this final profile is stable with respect to small perturbations of the initial conditions. Hence, we may expect (F2) holds for a wide class of solutions in higher dimensions, too.

\section{§3.3. Quenching problem}

In this subsection, we treat the following initial boundary value problem:

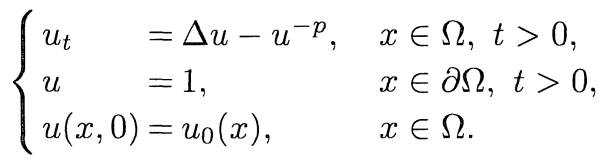


Here $\Omega \subset \mathbb{R}^{N}$ is a bounded domain. We assume that initial data $u_{0}$ is a smooth function such that $0<u_{0} \leq 1$. It is well known that the minimum of $u$ approaches zero as $t$ tends to a finite time $T$ provided $\Omega$ is sufficiently large. Moreover, as $t$ tends to $T, u_{t}$ tends to infinity. (See for instance [30], [14], [15], [25], [16]). This phenomenon is called quenching. This kind of phenomenon was first studied by Kawarada [27].

We assume that $N=1, p>1$, and $\Omega=(0, a)$ and $a>0$. We wish to approximate the quenching time $T$ by the solution of the following approximate problem:

$$
\left\{\begin{aligned}
\frac{d}{d t} u_{\jmath}^{n} & =\left(\Delta_{h} u^{n}\right)_{\jmath}-\left(u_{\jmath}^{n}\right)^{-p}, & & t>0,0<j<n, \\
u_{\jmath}^{n} & =1, & & j=1, n, t>0, \\
u_{\jmath}^{n}(0) & =\left(u_{0}^{n}\right)_{\jmath}, & & 0 \leq j \leq n .
\end{aligned}\right.
$$

Here, we set $h=a / n$ and $\left(\Delta_{h} u\right)_{\jmath}=\frac{u_{\jmath-1}-2 u_{\jmath}+u_{\jmath+1}}{h^{2}}$. For this approximate problem, we can prove the convergence in the quite same manner as in the previous subsections.

We define the functionals $J$ and $J_{n}$ as follows:

$$
J[u](t)=\int_{\Omega} u(x, t) \varphi(x) d x, \quad J_{n}\left[u^{n}\right](t)=\sum_{\jmath=0}^{n} u_{\jmath}^{n}(t) \varphi(j h) h .
$$

Here $\varphi(x)$ denotes the first eigenfunction of $-\Delta$ under homogeneous Dirichlet boundary condition. We have

$$
\lim _{t \rightarrow T} \frac{d}{d t} J[u](t)=-\infty
$$

This can be shown by the next proposition which is a result of Fila and Kawohl [14].

Proposition 3.8. We assume that the solution $u$ of the problem (3.15) quenches at $t=T$. Then for $a>\frac{1+p}{2}$ we have

$$
\lim _{t \rightarrow T} \int_{\Omega} u^{-\alpha} \varphi d x=\infty
$$

Let us assume that the initial data satisfies following:

$$
\left(\Delta_{h} u_{0}^{n}\right)_{\jmath}-\left(u_{0}^{n}\right)_{\jmath}^{-p} \leq 0 .
$$

This assumption implies that $\frac{d}{d t} u^{n} \leq 0$, hence we have

$$
\frac{d^{2}}{d t^{2}} J_{n}\left[u^{n}\right](t) \leq-\lambda \frac{d}{d t} J_{n}\left[u^{n}\right](t)
$$


So the assumptions (A1") and (A2) are satisfied. Hence we obtain the following result by virtue of Theorem 1.2 .

Theorem 3.4. We assume that initial data satisfies the condition (Q1) and the error of initial data is of order o(1). We also assume that the solution $u$ quenches in finite time $T$. Then for sufficiently large $n$ the approximate solution $u^{n}$ quenches in finite time $T_{n}$. Moreover, we have

$$
\lim _{n \rightarrow \infty} T_{n}=T \text {. }
$$

\section{$\S 4$. Continuous Dependence of the Blow-up Time}

As a direct consequence of our main results, we can obtain that the blowup time depends on the initial data continuously. In this section, we explain this result briefly.

Let us consider equations $(1.1)$ and $\left(1.1_{\mathrm{h}}\right)$ again. We set $F_{h} \equiv F, B_{h} \equiv$ $B, \Omega_{h} \equiv \Omega, x_{h} \equiv x, \Delta_{h} \equiv \Delta$, and $\nabla_{h} \equiv \nabla$ in approximate equations $\left(1.1_{\mathrm{h}}\right)$. We assume that there exists a functional $J$ which satisfies one of (A1), (A1'), $\left(\mathrm{A} 1^{\prime \prime}\right)$, and $\left(\mathrm{A} 1^{\prime \prime \prime}\right)$ and we also assume

(A3) if initial data $u_{0}^{h}$ converges to $u_{0}$ in some topology as $h$ tends to zero then the condition (A2) or $\left(\mathrm{A} 2^{\prime}\right)$ holds with $J_{h} \equiv J$.

Then we can obtain the continuity of blow-up time with respect to initial data, by one of our main theorems.

Theorem 4.1. We assume (A0), (A3), and one of (A1), $\left(\mathrm{A}^{\prime}\right),\left(\mathrm{A} 1^{\prime \prime}\right)$, $\left(\mathrm{A} 1^{\prime \prime \prime}\right)$ with $J_{h} \equiv J$. Then blow-up time of $(1.1)$ is continuous with respect to initial data in the same topology as in (A3).

In many cases, the condition (A3) will be easily verified by the continuous dependence of the solution on the initial data.

Let us consider the problem of Fujita type. If we assume $u_{0}^{h}$ converges to $u_{0}$ in $H^{1}$ topology, then the corresponding solutions $u^{h}(t)$ converges to the solution $u(t)$ in $H^{1}$ topology. This fact leads the condition (A2) and (A2') for $J[u](t)=\int_{\Omega} u^{2} d x$ and $G_{h}(t)=-\int_{\Omega}\left|\nabla u^{h}\right|^{2} d x+\frac{2}{p+1} \int_{\Omega}\left(u^{h}\right)^{p+1} d x$. Hence we obtain the following result.

Theorem 4.2. We assume that the solution of (3.4) with $f(u)=u^{p}$ blows up in finite time $T$ and $\left\|u_{0}-u_{0}^{h}\right\|_{H^{1}(\Omega)}=o(1)$ as $h$ tends to zero. We assume one of the following: 
1. $1<p<\frac{N+2}{N-2}$ or $-I(t)$ tends to infinity as $t$ tends to $T$.

2. $1<p<1+4 / N$ or $J[u](t)$ tends to infinity as $t$ tends to $T$.

Then $u^{h}(t)$, which is the solution starting from $u_{0}^{h}$, blows up in finite time $T_{h}$ and we have $\lim _{h \rightarrow 0} T_{h}=T$.

Remark 4.1. The continuous dependence of the blow-up time on the initial data for Fujita type problem has been already proved by Merle [32].

Remark 4.2. We note that we can also prove the continuous dependence of the blow-up time on the initial data for GCF.

\section{Appendix. Discrete Analogue of Super and Subsolution Method}

In this section, we explain a discrete analogue of super and subsolution method. By this method, we obtain some properties of semi-discrete solution and convergence between approximate solution and continuous one.

We consider the following parabolic equation with homogeneous Dirichlet boundary condition:

$$
u_{t}=a(x) \Delta u+f(u), \quad(x, t) \in \Omega \times(0, T) .
$$

Here we set $\Omega=(0,1)^{N}, a(x)$ is a positive smooth function, and $f$ is a smooth function. We note that we can treat the problem under periodic boundary condition similarly. For this problem, we introduce the semi-discrete problem:

$$
\left\{\begin{array}{llrl}
\dot{u}_{J}=a_{J}\left(\Delta_{h} u\right)_{J}+f\left(u_{J}\right), & & J \in \mathcal{K}, t>0, \\
u_{J}(t)=0, & & J \in \mathcal{K}_{0}, t>0, \\
u_{J}(0)=\varphi_{J}, & & J \in \mathcal{K} .
\end{array}\right.
$$

Here we use the same notation as in Subsection 3.2. We assume that $a_{J}>0$ for all $J \in \mathcal{K}$.

For this semi-discrete problem, we introduce the notion of super and subsolutions.

Definition A.1 Function $u^{n}=\left\{u_{J}(t)\right\}$ is supersolution of (A.1) if and only if $u_{J}(t)$ satisfies

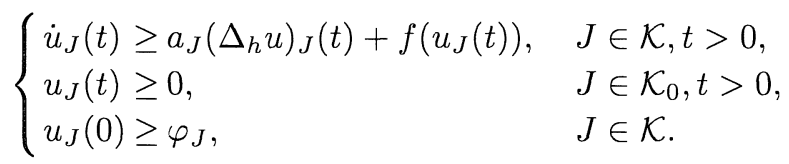


Function $u^{n}=\left\{u_{J}(t)\right\}$ is subsolution of (A.1) if and only if $u_{J}(t)$ satisfies opposite inequalities.

Theorem A.1. We assume that $u^{n}=\left\{u_{J}\right\}$ and $v^{n}=\left\{v_{J}\right\}$ are a supersolution of (A.1) and a subsolution of (A.1), respectively. Then for any $t \geq 0$ and $J \in \mathcal{K}$ we have $u_{J}(t) \geq v_{J}(t)$.

Proof. We fix $t_{0}$ such that $u_{J}$ and $v_{J}$ are bounded for $t$ in $\left[0, t_{0}\right]$. We set $w_{J}=u_{J}-v_{J}$. Then $w_{J}$ satisfies the following inequalities,

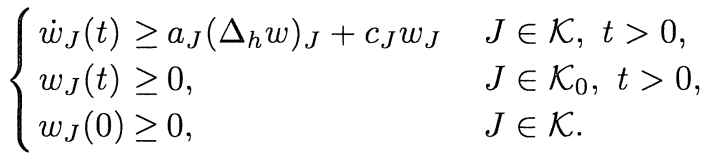

Here $c_{J}=\int_{0}^{1} f^{\prime}\left(s u_{\jmath}+(1-s) v_{\jmath}\right) d s$, which is bounded provided $u_{\jmath}$ and $v_{\jmath}$ are bounded. We set $W_{J}=e^{-k t} w_{J}(t)$ then $W_{J}$ satisfies,

$$
\dot{W}_{J} \geq a_{J}\left(\Delta_{h} W\right)_{J}+\left(c_{J}-k\right) W_{J}, \quad J \in \mathcal{K}, t \in\left[0, t_{0}\right] .
$$

For sufficiently large $k,\left(c_{J}-k\right)$ is negative.

If there exists a point $\left(J_{1}, t_{1}\right) \in \mathcal{K} \times\left[0, t_{0}\right]$ such that $w$ attains its negative minimum at this point. We can check that

$$
\dot{W}_{J_{1}}\left(t_{1}\right) \leq 0 \quad \text { and } \quad\left(\Delta_{h} W\right)_{J_{1}}\left(t_{1}\right) \geq 0 .
$$

So we have

$$
0 \geq \dot{W}_{J_{1}}\left(t_{1}\right) \geq\left(\Delta_{h} W\right)_{J_{1}}\left(t_{1}\right)+\left(c_{J}-k\right) W_{J}>0,
$$

which is a contradiction.

\section{References}

[1] Abia, L. M., López-Marcos, J. C. and Martínez, J., On the blow-up time convergence of semidiscretizations of reaction-diffusion equations, Appl. Numer. Math., 26 (1998), 399-414.

[2] Alvarez, L., Guichard, F., Lions, P. L. and Morel, J. M., Axioms and fundamental equations of image processing, Arch. Ratıonal Mech. Anal., 123 (1993), 199-257.

[3] Andrews, B., Evolving convex curves, Calc. Var., 7 (1998), 315-371.

[4] Angenent, S., On the formation of singularities in the curve shortening flow, J. Diff. Geom., 33 (1991), 601-633. 
[5] Angenent, S. and Gurtin, M. E., Multiphase thermodynamics with interfacial structure II, Evolution of an isothermal interface, Arch. Ratıonal Mech. Anal., 108 (1989), 323391.

[6] Angenent, S., Sapiro, G. and Tannenbaum, A., On the affine heat equation for nonconvex curves, J. Amer. Math. Soc., 11 (1998), 601-634.

[7] Angenent, S. and Velázquez, J. J. L., Asymptotic shape of cusp singularities in curve shortening, Duke Math. J., 77 (1995), 71-110.

[8] Bebernes, J. and Bricher, S., Final time blowup profiles for semilinear parabolic equations via center manifold theory, SIAM J. Math. Anal., 23 (1992), 852-869.

[9] Bebernes, J. and Eberly, D., Mathematıcal Problems from Combustıon Theory, SpringerVerlag, New York, 1989.

[10] Berger, M. and Kohn, R. V., A rescaling algorithm for the numerical calculation of blowing-up solutions, Comm. Pure Appl. Math., 41 (1988), 841-863.

[11] Bressan, A., Stable blow-up patterns, J. Diff. Eq., 98 (1992), 57-75.

[12] Budd, C. J., Huang, W. and Russell, R., Moving mesh methods for problems with blow-up, SIAM J. Scr. Comput., 17 (1996), 305-327.

[13] Chen, Y. G., Asymptotic behaviors of blowing-up solutions for finite difference analogue

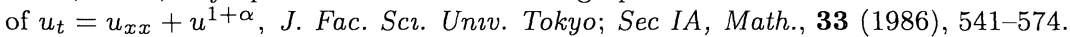

[14] Fila, M. and Kawohl, B., Asymptotic analysis of quenching problems, Rocky Mountaın J., 22 (1992), 563-577.

[15] Fila, M., Kawohl, B. and Levine, H. A., Quenching for quasilinear equations, Comm. PDE., 17 (1992), 593-614.

[16] Fillipas, S. and Guo, J. S., Quenching profiles for one dimensional semilinear heat equations, Quart. Appl. Math., 51 (1993), 713-729.

[17] Friedman, A. and McLeod, B., Blow-up of positive solutions of semilinear heat equations, Indrana Unvv. Math. J., 34 (1985), 425-447.

[18] Fujita, H., On the blowing up of solutions to the Cauchy problem $u_{t}=\Delta u+u^{1+\alpha}, J$. Fac. Scr. Unıv. of Tokyo, 13 (1966), 109-124.

[19] Gage, M. and Hamilton, R. S., The heat equation shrinking convex plane curves, $J$. Diff. Geom., 23 (1986), 69-96.

[20] Gage, M. E., On the size of the blow-up set for a quasilinear parabolic equation, Contemp. Math., 127 (1992), 41-58.

[21] Giga, Y., Solution for semilinear parabolic equations in $L^{p}$ and regularity of weak solutions of the Navier-Stokes system, J. Diff. Eq., 61 (1986), 186-212.

[22] - A bound for global solutions of semilinear heat equations, Comm. Math. Phys., 103 (1986), 415-421.

[23] Girão, P. M., Convergence of a crystalline algorithm for the motion of a simple closed convex curve by weighted curvature, SIAM J. Numer. Anal., 32 (1995), 886-899.

[24] Grayson, M. A., The heat equation shrinks embedded plane curves to round points, $J$. Diff. Geom., 26 (1987), 285-314.

[25] Guo, J. S., On the quenching behavior of the solution of a semilinear parabolic equation, J. Math. Anal. Appl., 151 (1990), 58-79.

[26] Ishiwata, T. and Tsutsumi, M., A numerical study of blow-up solutions to $u_{t}=u^{\delta}(\triangle u+$ $\mu$ u), Tran. Japan Soc. Indust. Appl. Math., 6 (1996), 219-231.

[27] Kawarada, H., On solutions of initial boundary problem for $u_{t}=u_{x x}+1 /(1-u)$, Publ. RIMS, Kyoto Unıv., 10 (1975), 729-736.

[28] Kimura, M., Accurate numerical scheme for the flow by curvature, Appl. Math. Lett., 7 (1994), 69-73.

[29] - Numerical analysis for moving boundary problems using the boundary tracking method, Japan J. Indust. Appl. Math., 14 (1997), 373-398.

[30] Levine, H. A., The phenomenon of quenching: A survey, in Trends in the theory and practıce of nonlınear analysıs, ed. V. Lakshmikantham, North Holland (1985), 275-286.

[31] - The role of critical exponents in blow-up theorems, SIAM Rev., 32 (1990), 262-288. 
[32] Merle, F., Solution of a nonlinear heat equation with arbitrarily given blow-up points, Comm. Pure Appl. Math., 45 (1992), 263-300.

[33] Mikula, K., Solution of nonlinear curvature driven evolution of plane convex curves, Appl. Num. Math., 23 (1997), 347-360.

[34] Mikula, K. and Ševčovič, D., Evolution of plane curves driven by a nonlinear function of curvature and anisotropy, preprint.

[35] Nakagawa, T., Blowing up of a finite difference solution to $u_{t}=u_{x x}+u^{2}$, Appl. Math. Optrm., 2 (1976), 337-350.

[36] Nakagawa, T. and Ushijima, T., Finite element analysis of the semilinear heat equation of blow-up type, Topıcs Numer. Anal., 3 (1977), 275-291.

[37] Samarskii, A. A., Galaktionov, V. A., Kurdyumov, S. P. and Mikhailov, A. P., Blow-up in quasılınear parabolıc equatıons, Walter de Gruyter, 1995.

[38] Sapiro, G. and Tannenbaum, A., On affine plane curve evolution, J. Funct. Anal., 119 (1994), 79-120.

[39] Straughan, B., Explosive Instabilities in Mechanics. Springer-Verlag, Berlin, 1998.

[40] Ushijima, T. K. and Yazaki, S., Convergence of a crystalline algorithm for the motion of a closed convex curve by a power of curvature $V=K^{\alpha}$, SIAM J. Num. Anal., 37 (2000), 500-522.

[41] - Convergence of a crystalline algorithm for the motion of a closed convex curve by a power of curvature $V=K^{\alpha}$, in GAKUTO Internat. Ser. Math. Scı. Appl., 12, Adv. Numer. Math.; Proceedings of the Fourth Japan-Chına Joint Semınar on Numerical Mathematıcs, ed. Kawarada, H., Nakamura, M. and Shi, Z., Gakkōtosho (1999), 261270.

[42] Velázquez, J. J. L., Blow up for semilinear parabolic equations, in Recent Advances ın Partıal Differentıal Equatıons, ed. Herrero, M. A. and Zuazua, E., Masson (1994), 131-145. 